\title{
Air sampling and analysis of indoor fungi: a critical review of passive (non- activated) and active (activated) sampling
}

\author{
Spyridon Efthymiopoulos ${ }^{\mathrm{a}, \mathrm{b}, *}$, Yasemin Didem Aktas ${ }^{\mathrm{a}, \mathrm{b}}$, Hector Altamirano-Medina ${ }^{\mathrm{b}, \mathrm{c}}$ \\ a Department of Civil Environmental and Geomatic Engineering (CEGE),UCL, London, UK \\ $b$ UK Centre for Moisture in Buildings (UKCMB), London, UK \\ c Institute of Environmental Design and Engineering (IEDE),UCL, London, UK
}

\begin{abstract}
For over a century, researchers have been concerned with what testing protocols to assess indoor fungi growth were most reliable. To that end, various sampling and analysis methods were developed. However, to present no testing procedure has been standardised. The vast number of different fungi species, the differences in their biological properties and their implications on the occupants' health and building fabric can make the decision-making process for an appropriate assessment protocol challenging. This research aims to make a critical review of passive (or nonactivated) and active (or activated) sampling and emphasize potential errors while interpreting results obtained from passive or active protocols. Peer-review under the responsibility of the organizing committee of the ICMB21.
\end{abstract}

Keywords: Passive (non-activated) sampling; Active (activated) sampling; Indoor Fungi; Mould assessment; Mould testing

\section{Introduction}

Indoor fungal growth can cause health implications to the occupants [1], disturb their comfort and well-being, and lead to damage of the building's fabric [2]. Therefore, it is of critical importance to be able to measure the extent of fungal growth correctly in a given indoor environment. Through testing protocols, researchers aim to determine the conditions under which fungi flourish and the consequences of fungal contamination to the occupants' health, and to assess whether the property is in need of remediation [3]. However, though many protocols have been implemented, no indoor fungal testing protocol has yet been standardised. The present study aims to underline the importance of passive and active air sampling for indoor fungal testing.

\section{Indoor air sampling for fungal testing}

\subsection{Passive (non-activated) and active (activated) sampling: Terminology}

The terms passive and active sampling may be sometimes unclear. According to Aktas et al. [4,5], Heinsohn [3] and Swaebly and Christensen [6] active (also known as aggressive or activated) sampling involves the collection of particles after the disturbance of the air through artificial means, while passive (also known as non-aggressive or non-activated) sampling is used to describe the collection of particles from still air. Other researchers have used the passive and active terms as means to discriminate between air sampling through gravitational settling (e.g. sedimentation) and air sampling through the creation of an artificial airflow (e.g. impaction, liquid impingement, filtration and electrostatic precipitation) [7-9]. It can be argued that in both cases, the two terms attempt to define whether samples are collected from still or mixed air, respectively. However, there is a distinct difference which relies on the time at which the air is being mixed. In the first case, the terms have been used to define whether the air has been mixed prior to sampling, while on the second case the terms describe whether the air's steadiness is disturbed during sampling. Therefore, within the literature, two different uses of the terms should not be confounded. In this paper, the terms passive (non-activated) and active (activated) sampling will be used to point to the air mixing levels prior to testing.

\subsection{Passive (non-activated) vs active (activated) sampling: How they manifest in the results}

According to the definitions given earlier, passive (non-activated) and active (activated) sampling refer to still and mixed air. However, stillness or mixed-ness of air can be defined differently, which is a barrier to adopting these terms for repeatable testing protocols. For example, Rylander [10] has shown that the mould levels measured in a room increased as the level of activity inside the room prior to testing increased. A similar trend has been identified by Flannigan [11], who indicated that the human activity in a restaurant affected the fungal viable counts. Therefore, the level and nature of activity within a given indoor space is influential

\footnotetext{
* Spyridon Efthymiopoulos. +44 7585 377455. spyros.efthymiopoulos.18@ucl.ac.uk
} 


\section{ICMB21}

on the testing outcome and unless the conditions under which the sampling is performed are well defined, classifying a protocol as non-activated and activated does little to ensure its reproducibility.

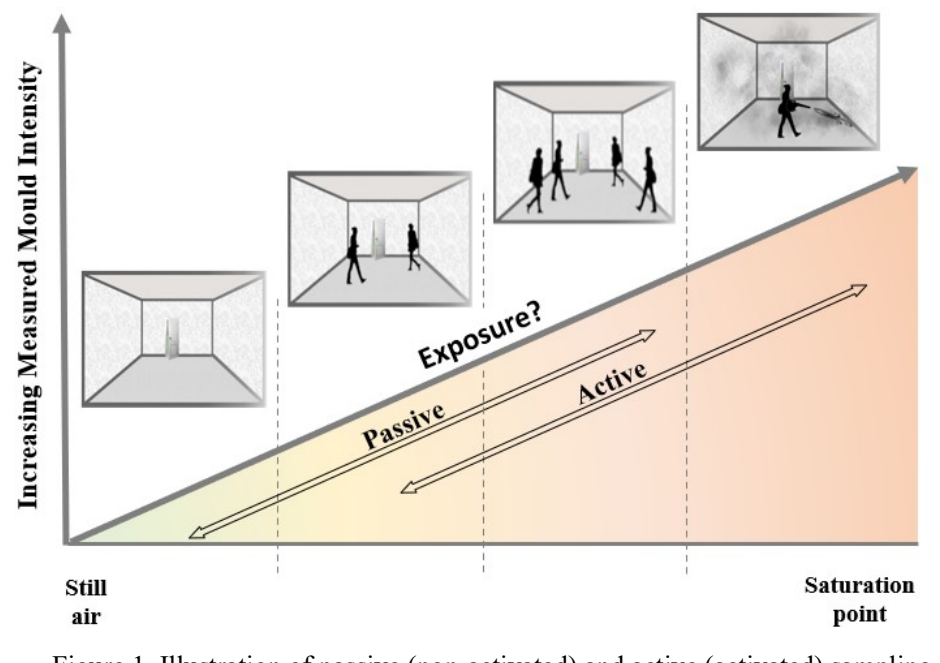

Figure 1. Illustration of passive (non-activated) and active (activated) sampling

It is important to mention that a review of the literature indicates a prevalence of passive (non-activated) readings in fungi assessment-related studies. Researchers have often turned to passive (non-activated) sampling under the claim that active (activated) protocols are not reproducible [3], however it was also claimed that the passive (non-activated) protocols underestimate the fungal biomass, which can be critical in studies estimating exposure [4-5]; in any case, many studies fail to sufficiently detail the conditions under which these protocols were implemented or report limited control over the testing conditions. Furthermore, to present, there is limited literature on the effect of well-defined sampling protocols to the readings, while there are no widely accepted passive (non-activated)/active (activated) standards to ensure replicability of the testing methods. Hence, lack of a welldefined passive (non-activated)/active (activated) protocol, may result to the acquisition of sampling readings that may not be comparable to corresponding ones from other testing sites.

\section{Discussion}

Researchers should give attention to the conditions under which the testing is carried out. Passive (non-activated) and active (activated) readings do differ [5]. In the case of active (activated) sampling, disturbing the air's stillness may increase the concentration of airborne fungal fragments and spores, and thus could lead to the identification of fungi that could otherwise be undetectable. However, without defining the actual air mixing levels and detailing how these manifest in the test outcomes, the classification of sampling into passive (non-activated) and active (activated) protocols cannot provide evidence of reproducibility of the implemented testing method. For the purpose of developing rigorous and reproducible protocols able to give answers sought by testing, it is of utmost importance that research on the effect of passive (non-activated) and active (activated) protocols to the air sampling readings is further developed.

\section{References}

[1] World Health Organization Europe (2009) 'Dampness and mould', WHO guidelines for indoor air quality: dampness and mould, pp. 1-228.

[2] Anderson, A. A. (1958) 'New sampler for the collection, sizing, and enumeration of viable airborne particles', U. S. Army Chemical Corps Proving Ground, 76(904), pp. 471-484.

[3] Heinsohn, P. A. (2007). Conducting building mold investigations. In C. S. Yang \& P. A. Heinsohn (Eds.), Sampling and Analysis of Indoor Microorganisms. [4] Aktas, Y. D., Altamirano-Medina, H., Ioannou, I., May, N., \& D’Ayala, D. (2018). Indoor Mould Testing and Benchmarking: Public Report. (February), 116.

[5] Aktas, Y. D. et al. (2018) 'Surface and passive/active air mould sampling: A testing exercise in a North London housing estate', Science of the Total Environment, 43, pp. 1631-1643.

[6] Swaebly, M. A. and Christensen, C. M. (1952) 'Molds in house dust, furniture stuffing, and in the air within homes', Journal of Allergy, $23(4)$, pp. 370-374. [7] Mainelis, G. (2019) 'Bioaerosol sampling: Classical approaches, advances, and perspectives', Aerosol Science and Technology. Taylor \& Francis, 0(0), pp. $1-24$.

[8] Amato, P., et al. (2018). Sampling Techniques. In A.-M. Delort \& P. Amato (Eds.), Microbiology of Aerosols (1st ed.). Hoboken: John Wiley \& Sons. [9] Grinshpun, S. A. (2010) Biological Aerosols. In Aerosols - Science and Technology. Edited by I. Agranovski. Weinheim: Wiley-VCH.

[10] Rylander, R. (2015) ' $\beta$-N-Acetylhexosaminidase (NAHA) as a Marker of Fungal Cell Biomass - Storage Stability and Relation to $\beta$-Glucan', International Journal of Environmental Monitoring and Analysis, 3(4), p. 205.

[11] Flannigan, B. (1992) Indoor microbiological pollutants-sources, species, characterisation and evaluation. In Chemical, Microbiological, Health and Comfort Aspects of Indoor Air Quality- State of the Art in SBS (Edited by Knoppel, H. and Wolkoff, P.), pp. 73398. Kluwer, Dordrecht.

\section{Acknowledgments}

This study has been carried out as part of the EPSRC-DTP-CASE (EP/R513143/1) project where POLYGON UK was the industry sponsor. We are greatly thankful to both funding bodies. 